\title{
Adaptação Transcultural de Escala de Auto-Estima para Adolescentes
}

\author{
Cross-Cultural Adaptation of Self-Esteem Scale for Adolescents
}

\author{
Joviana Q. Avanci ${ }^{*}{ }^{*}$, Simone G. Assis ${ }^{a}{ }^{b}$, Nilton César dos Santos ${ }^{a} \&$ Rachel V. C. Oliveira ${ }^{a}$ \\ ${ }^{a}$ Fundação Oswaldo Cruz, Rio de Janeiro, Brasil \\ ${ }^{b}$ Escola Nacional de Saúde Pública, Rio de Janeiro, Brasil
}

\begin{abstract}
Resumo
O ar tigo propõe a padronização da escala de auto-estima de Rosenberg (1956/1989) para adolescentes residentes de um bairro urbano do Rio de Janeiro (São Gonçalo), através do estudo de adaptação transcultural (Herdman, Fox-Rushby \& Badia, 1998). A amostra foi composta por 266 adolescentes escolares das $7^{\mathrm{a}} / 8^{\mathrm{a}}$ séries do Ensino Fundamental e $1^{\circ} / 2^{\circ}$ anos do Ensino Médio da rede pública e particular do referido município. As seguintes equivalências foram avaliadas: conceitual e de itens, semântica (significado referencial e geral), de mensuração (confiabilidade teste-reteste, consistência interna, análise fatorial e validade de construto), operacional e funcional. Foram encontrados bons resultados para equivalência semântica, o alfa de Cronbach foi de 0.68, Kappa predominantemente moderado e regular, e a análise fatorial proposta é de duas estruturas fatoriais (baixa e alta auto-estima). Na validade de construto, a escala correlacionou-se significativa e positivamente com apoio social, e inversamente com vitimização de violência psicológica, violência ocorrida entre pais e entre os irmãos. Os resultados indicam a aplicabilidade da escala na população de referência, sugerindo a necessidade do desenvolvimento de outros trabalhos em amostras distintas.

Palavras-chave: Auto-estima; Escala; Confiabilidade; Validade.
\end{abstract}

\begin{abstract}
The article proposes a cross-cultural adaptation (Herdman, Fox-Rushby \& Badia, 1998) of "Rosenberg SelfEsteem Scale" for adolescents who live in an urban neighborhood of Rio de Janeiro (São Gonçalo). The sample was composed of 266 adolescents, students of the 7th/ 8th grade of Elementary School and of the 1st/2nd grade of High School, of public and private schools of São Gonçalo/RJ. The following equivalences were evaluated: conceptual and itens equivalences, semantic equivalence (referential and general meaning), measurement equivalence (test-retest reliability, internal consistency, factorial analysis and construct validity) and operational and functional equivalences. Good results were obtained for semantics equivalence, alpha of Cronbach was of 0.68 , the Kappa was moderate and regular, and the factorial analysis proposed two structures of factors (low and high self-esteem). Construct validity showed significant positive correlation with social support and negative correlation with psychological abuse, violence between parents and brothers. The results indicate the applicability of the scale in a reference population, suggesting the necessity to develop others studies in distinct samples.

Keywords: Self-esteem; Scale; Reliability; Validity.
\end{abstract}

No Brasil (Gobitta, 2000; Hutz, 2000) e em vários outros países, a temática da auto-estima é pouco abordada cientificamente. Palavra fácil na psicologização das relações humanas, o tema da auto-estima se tornou popularizado por livros de auto-ajuda e pelo senso comum, o que acarreta dificuldades conceituais e metodológicas. Se por um lado, a propagação "superficial" desse conceito traz

\footnotetext{
* Endereço para correspondência: Avenida Brasil, 4036, sala 700, Manguinhos, Rio de Janeiro, RJ, 21040-631. E-mail joviana@claves.fiocruz.br

A pesquisa recebeu suporte do Conselho Nacional de Desenvolvimento Científico e Tecnológico/CNPq. Também contou com bolsista da Fundação de Amparo à Pesquisa do Rio de Janeiro - FAPERJ, e do Programa PIBIC do CNPq/ Fiocruz. Um especial agradecimento a Dra. Mônica Gobitta, Dr. Claúdio Hutz e Dra. Gabriela Crenzel que contribuíram com o processo de adaptação apresentada.
}

dificuldades, requerendo esforço na consolidação científica de algo já inscrito no senso comum; por outro, traz vantagens, uma vez que é uma temática já inscrita no imaginário social.

Por auto-estima entende-se a avaliação que o indivíduo faz de si mesmo. Expressa uma atitude de aprovação ou de repulsa de si e engloba o auto-julgamento em relação à competência e valor. É o juízo pessoal de valor revelado através das atitudes que um indivíduo tem consigo mesmo, sendo uma experiência subjetiva acessível às pessoas através de relatos verbais e comportamentos observáveis (Coopersmith, 1967). Pode ser avaliada segundo níveis: baixo, médio e alto. A baixa auto-estima caracteriza-se pelo sentimento de incompetência, de inadequação à vida e incapacidade de superação de desafios; a alta expressa um sentimento de confiança e competência; e a média flutua 
entre o sentimento de adequação ou inadequação, manifestando essa inconsistência no comportamento (Branden, 2000; Rosenberg, 1956/1989).

Estudos que avaliam a importância da auto-estima proliferam nos países desenvolvidos, destacando-se dentre os indicadores de saúde mental e nas análises sociais de crescimento e progresso (Mruk, 1995). A violência familiar, o abuso de drogas, a gravidez precoce, o fraco desempenho escolar, a delinqüência, o suicídio, as agressões escolares, a depressão e a prostituição são alguns dos problemas ditos contemporâneos associados à baixa auto-estima (Assis \& Avanci, 2003; Dourado, 1984; Gomes, 1994; Mecca, Smelser \& Vasconcellos, 1989; Rosenberg, 1956/ 1989; Tamayo \& Cunha, 1983).

Embora a importância da auto-estima para o bem-estar social e individual seja reconhecida internacionalmente, no Brasil, há escassez de estudos sobre a temática, especial-mente em bases populacionais. Um dos obstáculos a uma abordagem epidemiológica da auto-estima é a falta de instrumentos diagnósticos consolidados cientificamente em amostras brasileiras, o que impede o conhecimento desse "atributo" em contextos de investigação.

Pretendendo contribuir com esse panorama, o objetivo desse trabalho é apresentar estratégias e resultados da adaptação transcultural da escala de auto-estima concebida por Rosenberg (1956/1989) em língua inglesa, sugerindo uma versão em português. Esse instrumento já vem sendo utilizado no meio científico (Giacomoni, 2002; Hutz, 2000; Souza \& Ferreira, 2005), no entanto algumas importantes etapas do processo de avaliação da adaptação transcultural não foram ainda contempladas.

De modo geral, a escala em estudo avalia a atitude e o sentimento positivo ou negativo por si mesmo. Engloba questões de satisfação pessoal, auto-depreciação, percepção de qualidades, competência, orgulho por si, auto-valorização, respeito e sentimento de fracasso. Para realizar a adaptação transcultural do instrumento estudado, optou-se pelo modelo proposto por Herdman, Fox-Rushby e Badia (1998) e difundido por pesquisadores brasileiros (Hasselmann \& Reichenheim, 2003; Moraes \& Reichenheim, 2002). Todas as fases de adaptação propostas pelos autores foram realizadas, as quais englobam as seguintes equivalências: conceitual, de itens, semântica, operacional e de mensuração e a funcional entre o instrumento original na língua inglesa e uma versão em português. Apresenta também a análise da validade de construto através da eleição de variáveis, cujo conhecimento teórico e empírico demonstra associação significativa com a auto-estima. A partir disso, pretende-se que a escala estudada possa ser refinada e aplicada em estudos epidemiológicos, o que contribuirá para o conhecimento da questão no país.

\section{Método}

A escala de auto-estima de Rosenberg foi selecionada mediante um levantamento bibliográfico em bases de dados nacionais e internacionais sobre instrumentos que avaliam o construto de interesse. Os seguintes fatores foram decisivos para a sua escolha: (a) faixa etária da amostra original condizente à população estudada; (b) adequação teórica do tema à cultura brasileira; (c) consistente processo de criação da escala, realizado através da aplicação com 5024 estudantes de escolas públicas de Nova York; (d) bons coeficientes psicométricos obtidos originalmente (coeficiente de confiabilidade de 0.92 ) e em outros trabalhos (Hutz, 2000; Giacomoni, 2002); (e) validade de construto através da associação positiva com escalas que avaliam depressão e sintomas psicossomáticos (Rosenberg, 1956/1989; Roberts \& Kendler, 1999); (f) é um dos instrumentos mais utilizados em trabalhos internacionais para aferir a auto-estima (Hutz, 2000); (g) praticidade da escala, com número de itens inferior as outras pesquisadas, a exemplo da proposta por Coopersmith (1967).

A escala em estudo é tipo Likert, constituída por dez questões fechadas, com as seguintes opções de resposta concordo totalmente, concordo, discordo e discordo totalmente, onde cada item de resposta varia de 1 a 4 pontos. Quanto maior o escore, maior o "nível" da auto-estima. Todos os itens foram submetidos ao processo de adaptação transcultural ora apresentado. A aplicação da escala foi realizada de forma anônima e autopreenchível.

\section{Participantes}

Participaram 266 alunos de 11 a 19 anos de idade, de 2 escolas públicas (rede municipal e estadual) e 1 particular do município de São Gonçalo, sendo estudantes de $7^{\mathrm{a}}$ e $8^{\mathrm{a}}$ séries do Ensino Fundamental e $1^{\mathrm{o}}$ e $2^{\circ}$ ano do Ensino Médio. São Gonçalo é um município que integra a Região Metropolitana do Estado do Rio de Janeiro, sendo o segundo maior município do estado em termos populacionais e o décimo quinto do país, com uma população de 889.828 habitantes vivendo em área urbanizada. Trinta e cinco por cento da população está na faixa de o a 19 anos de idade.

A amostragem do grupo estudado foi executada de forma não-probabilística, seguindo apenas o critério de escolha do pesquisador, uma vez que o objetivo dessa etapa não consistia na representatividade, mas sim no aprimoramento da escala, através, inclusive, da avaliação dos coeficientes psicométricos. A escolha das escolas participantes dessa etapa baseou-se na facilidade de inserção dos pesquisadores nessas unidades escolares.

\section{Procedimentos}

A escala foi aplicada por uma equipe de 4 entrevistadores, que foram rigorosamente treinados, durando em média 60 minutos. Os dados foram processados no programa Epi- 
info 6.0, encontrando-se um baixo percentual de erros de digitação (inferior a $1 \%$ do total de questões).

Equivalência Conceitual $e$ de Itens: a conceitual avalia a equivalência do construto na cultura original em comparação a alvo. A de itens avalia se os tópicos que compõem a escala estimam os mesmos domínios e são relevantes nas duas culturas (Herdman et al., 1998).

Essas equivalências foram realizadas através da discussão com dois experts, psicóloga e médica epidemiologista, com experiência na área de desenvolvimento infanto-juvenil. A discussão abordou os seguintes aspectos: verificação de itens que não tivessem a mesma conotação do termo original e na população alvo; adequação conceitual e de diagnóstico em ambas as culturas; capacidade dos itens em representar o domínio abarcado pelo instrumento; e adequação conceitual em relação ao perfil sócio-econômico e de faixa etária da população alvo.

Equivalência Semântica: refere-se a tradução do instrumento original e objetiva alcançar efeitos similares em respondentes de culturas distintas (Herdman et al., 1998). Os dez itens foram avaliados em quatro etapas: (a) duas traduções do instrumento original em inglês para o português, realizadas de forma independente (uma delas foi realizada por Hutz - comunicação pessoal, expert Psicologia); (b) essas duas traduções foram retraduzidas para o português por outros dois tradutores, também independentemente; (c) avaliação formal por um quinto tradutor, conhecedor da cultura americana e da área de Saúde Mental, a quem foi concebida a tarefa de avaliar a equivalência entre o original e cada uma das retraduções, sob a perspectiva do significado geral e referencial; (d) apreciação da equivalência semântica propriamente dita (Moraes, 2001). Os tradutores e retradutores que participaram do processo foram profissionais de nível superior, com graduação em letras (um em psicologia) e com especialização na língua inglesa.

Para a apreciação da terceira etapa, um formulário específico foi elaborado, onde dispunha aleatoriamente a versão original e as retraduções. Através da comparação das versões produzidas e da original, o avaliador apreciou duas categorias: o significado referencial (corresponde à concordância em termos de tradução literal entre um item original e o mesmo retraduzido, avaliado pela Visual Analogue Scale em que a equivalência entre pares é julgada de o a 100\%) e o significado geral (corresponde à concordância mais ampla, em termos de articulação de idéias e impacto entre um item original e sua retradução, avaliada através de quatro níveis de equivalência: inal terado, pouco alterado, muito alterado ou completamente alterado). Essa avaliação foi feita de forma "cega", dificultando a identificação do item original e do que sofreu tradução/ retradução. Houve um encontro com o avaliador dessa etapa, a fim de conhecer as justificativas para as suas pontuações, o que muito contribuiu para a consolidação do instrumento final (Moraes, 2001).
$\mathrm{Na}$ última etapa, houve uma discussão com experts da área de saúde mental, que avaliaram as etapas anteriores e definiram uma versão, que ora incorporou itens oriundos de uma das duas versões trabalhadas, ora optou por modificações mais pertinentes. Os itens foram modificados ou substituídos com a finalidade de propor uma versão final a ser subseqüentemente posta a teste.

Equivalência Operacional: objetiva manter características operacionais do universo original, propiciando maior confiabilidade e validade do instrumento. Foi apreendida através de medidas aplicadas antes e durante a aplicação da escala, tais como: mesmo número de questões e opções de resposta utilizadas no instrumento original; formato auto-preenchível acompanhado por aplicadores treinados, com tempo médio de 60 minutos por turma; treinamento dos aplicadores, tendo como base a elaboração de um manual instrutivo para orientar quanto ao preenchimento dos alunos.

Equivalência de Mensuração: consiste em avaliar as medidas de confiabilidade e validade da versão do instrumento, comparando-as com as encontradas no instrumento original (Moraes, 2001). A confiabilidade intra-observador, a consistência interna, a estrutura de fatores e aspectos da validade de construto foram apreciadas.

$\mathrm{Na}$ confiabilidade intra-observador, os adolescentes responderam o instrumento em dois momentos distintos, num intervalo de 7 a 10 dias. Os alunos foram orientados a colocar as iniciais do seu nome e a data de nascimento para facilitar essa comparação e evitar a identificação do respondente. Foi estimada pelo coeficiente de correlação intraclasse (ICC) para testar a concordância do instrumento em dois momentos. Utilizou-se também a estatística do kappa (kw), cuja interpretação seguiu a proposta de Shrout (1998). A concordância foi classificada como: substancial $(\mathrm{k}=0,81-1,0)$, moderada $(\mathrm{k}=0,61-0,81)$, discreta $(\mathrm{k}=$ $0,41-0,60)$, fraca $(k=0,10-0,40)$ e ausente $(k<0,1)$.

A avaliação da consistência interna baseou-se no estimador alfa de Cronbach (Streiner \& Norman, 1995). Na análise fatorial, o método de extração utilizado foi o Método das Componentes Principais, rotação varimax e extraindo-se os autovalores maiores que um (Streiner \& Norman, 1995).

A validade de construto foi examinada através da aplicação, na amostra selecionada, de instrumentos psicométricos elegíveis e pertinentes ao quadro teórico subjacente. As escalas estudadas foram selecionadas por evidenciarem associações com o construto de interesse, além de terem sido insistentemente analisadas e verificadas suas propriedades, indicando adequação e eficiência (Assis \& Avanci, 2003; Baumeister Smart, Boden, 1996; Kaplan, Pelcovitz \& Labruna, 1999). As seguintes escalas foram estudadas quanto à correlação, através do coeficiente de Pearson:

1. Escala de Apoio Social: composta por 19 itens, constituída por cinco dimensões: (a) emocional (apoio recebido através da confiança, da disponibilidade em ouvir, 
compartilhar preocupações/medos e compreender problemas), (b) de informação (através do recebimento de sugestões, bons conselhos, informação e conselhos desejados), (c) material (ajuda se ficar de cama, levar ao médico, preparar refeições e ajudar nas tarefas diárias se ficar doente), (d) afetiva (demonstração de afeto e amor, dar um abraço e amar) e (e) de interação positiva (divertir-se, relaxar, fazer coisas agradáveis e distrairse). Cinco escores são obtidos para cada dimensão através da proporção da soma dos itens com as seguintes opções de resposta: nunca, raramente, às vezes, quase sempre e sempre (Chor, Griep, Lopes \& Faerstein, 2001). No presente trabalho, verificou-se alfa de Cronbach de 0.93, ICC em torno de 0.65 para cada tipo de apoio investigado e kappa variando do regular ao moderado (Assis \& Avanci, 2003).

2. Violência Psicológica: avalia experiências vividas pelo jovem em que uma pessoa significativa diminuiu suas qualidades, suas capacidades, desejos, emoções e/ou que cobrou-o excessivamente (Avanci, Assis, Santos \& Oliveira, 2005). Foi adaptada transculturalmente por Avanci, Assis, Santos e Oliveira (2005), sendo constituída por 18 itens com opções de resposta que variam do nunca ao sempre. Neste trabalho, obteve-se alfa de Cronbach de 0.93 e ICC de 0.82 (Assis \& Avanci, 2003).

3. Violência Entre Irmãos e Entre Pais: composto por três itens para cada forma de violência. Investigaram as brigas entre os irmãos e entre os pais a ponto de se machucarem, xingarem ou humilharem um ao outro. Em relação aos irmãos, o alfa de Cronbach obtido foi de 083, na análise fatorial um fator foi gerado com carga superior a 0.80, ICC de 0.6 e kappa moderado. $\mathrm{Na}$ violência entre os pais, também foi gerado um fator com grau de explicação de $65.7 \%$ e ICC de 0.68 (Assis \& Avanci, 2003).

Questões Éticas: obteve-se autorização da Secretaria Municipal e Estadual de Educação de São Gonçalo. A direção das escolas e os alunos assinaram Consentimentos Livres e Esclarecidos, conforme preconizado na Resolução 196/ 96 do Conselho Nacional de Saúde, que normaliza as pesquisas com seres humanos. A pesquisa foi aprovada pelo Comitê de Ética em Pesquisa da Escola Nacional de Saúde Pública/FIOCRUZ.

\section{Resultados}

Equivalência Conceitual e de Itens

Nenhum item foi excluído da escala original, já que, a partir de uma revisão bibliográfica sobre o tema, os especialistas concluíram que o conceito subjacente utilizado para avaliar a auto-estima era pertinente à cultura brasileira. Foram também aceitos os critérios citados como pontos de discussão pelos especialistas.

Pretendendo tornar a versão em português mais adequada à população estudada, dois itens da escala foram mais discutidos pelos pesquisadores. Uma deles foi a do item de número 2 onde lia-se "às vezes eu acho que eu não sou bom na verdade”, o qual foi aceita a proposta por Hutz "às vezes eu acho que não presto para nada", já que essa é mais próxima da língua coloquial brasileira, que refere-se a um sentimento depreciativo de si mesmo, aspecto fundamental da auto-estima. Da mesma forma, o item de número 9 refere-se a "no todo eu me sinto inclinado a sentir que eu sou um fracasso", no qual o termo no todo foi substituído por no geral, expressão que denota maior amplitude na avaliação de si mesmo. Essas discussões também estiveram presentes na análise da equivalência semântica.

A maior preocupação e esforço dos pesquisadores nessa etapa foram optar por termos que se aproximassem da experiência e do sentimento de si mesmo, sem deixar de lado aspectos que causassem desconforto para o adolescente, o que poderia interferir em sua resposta ao instrumento.

\section{Equivalência Semântica}

Como pode ser observado na Tabela 1, no significado geral os avaliadores consideraram a grande parte dos itens (70\%) como pouco alterados e inalterados nas traduções e retraduções realizadas. Percentual semelhante (70\%) é encontrado na avaliação do significado referencial, onde a maioria dos índices foi superior a $80 \%$. Três itens se mostraram mais problemáticos nas avaliações, o de número 2 , 3 e 9, os quais foram bastante discutidos na avaliação da equivalência conceitual e de itens. Considerando pelo menos um avaliador, esses itens foram os que apresentaram menor percentual de concordância seja no sentido geral e/ou referencial. 
Avanci, J. Q., Assis, S. G., Santos, N. C. dos. \& Oliveira, R. V. C. (2007). Adaptação Transcultural de Escala de Auto-Estima para Adolescentes.

Tabela 1

Etapa de Equivalência Semântica da Adaptação Transcultural da Escala de Auto-Estima de Rosenberg.

\begin{tabular}{|c|c|c|c|c|c|c|c|c|}
\hline \multirow{2}{*}{$\begin{array}{l}\text { Formulário } \\
\text { Original } \\
\text { (Inglês) }\end{array}$} & \multicolumn{3}{|c|}{ Versão 1} & \multirow{2}{*}{$\begin{array}{r}{ }^{*} \text { S } \\
\mathrm{G} \\
\mathrm{A} 1\end{array}$} & \multicolumn{2}{|c|}{ Versão 2} & *SR & *** $\mathrm{S}$ \\
\hline & Tradução & Retradução & $\mathrm{A} 1$ & & Tradução & Retradução & $\mathrm{A} 1$ & $\begin{array}{l}\text { G } \\
\text { A1 } 1\end{array}$ \\
\hline $\begin{array}{l}\text { 1. On the } \\
\text { whole, I am } \\
\text { satisfied with } \\
\text { myself }\end{array}$ & $\begin{array}{l}\text { 1. No conjunto, } \\
\text { eu estou } \\
\text { satisfeito } \\
\text { comigo }\end{array}$ & $\begin{array}{l}\text { 1. As a whole, } \\
\text { I am satisfied } \\
\text { with myself }\end{array}$ & $100 \%$ & $\mathrm{PA}$ & $\begin{array}{l}\text { 1. No todo, estou } \\
\text { satisfeito (a) } \\
\text { comigo mesmo (a) }\end{array}$ & $\begin{array}{l}\text { 1. As a whole, } \\
\text { I am pleased } \\
\text { with myself }\end{array}$ & $100 \%$ & PA \\
\hline $\begin{array}{l}\text { 2. At times } \\
\text { I think I am } \\
\text { no good at all }\end{array}$ & $\begin{array}{l}\text { 2. Às vezes eu } \\
\text { acho que não } \\
\text { presto para nada }\end{array}$ & $\begin{array}{l}\text { 2. Sometimes } \\
\text { I feel myself } \\
\text { useless }\end{array}$ & $100 \%$ & PA & $\begin{array}{l}\text { 2. Às vezes eu acho } \\
\text { que eu não sou } \\
\text { bom na verdade }\end{array}$ & $\begin{array}{l}\text { 2. Sometimes I } \\
\text { think I am not so } \\
\text { good in fact }\end{array}$ & $50 \%$ & MA \\
\hline $\begin{array}{l}\text { 3. I feel that } \\
\text { I have a } \\
\text { number of } \\
\text { good qualities }\end{array}$ & $\begin{array}{l}\text { 3. Eu acho que } \\
\text { eu tenho várias } \\
\text { boas qualidades }\end{array}$ & $\begin{array}{l}\text { 3. I think I have } \\
\text { lots of good } \\
\text { qualities }\end{array}$ & $80 \%$ & $\mathrm{PA}$ & $\begin{array}{l}\text { 3. Eu sinto que } \\
\text { tenho inúmeras } \\
\text { qualidades (boas, } \\
\text { positivas) }\end{array}$ & $\begin{array}{l}\text { 3. I feel I have } \\
\text { innumerable } \\
\text { qualities (good } \\
\text { and positive } \\
\text { ones) }\end{array}$ & $70 \%$ & MA \\
\hline $\begin{array}{l}\text { 4. I am able to } \\
\text { do things as } \\
\text { well as most } \\
\text { other people }\end{array}$ & $\begin{array}{l}\text { 4. Eu acho que } \\
\text { sou capaz de } \\
\text { fazer as coisas } \\
\text { tão bem quanto } \\
\text { a maioria das } \\
\text { pessoas }\end{array}$ & $\begin{array}{l}\text { 4. I think I can do } \\
\text { things so well as } \\
\text { majority of people }\end{array}$ & $80 \%$ & $\mathrm{PA}$ & $\begin{array}{l}\text { 4. Eu sou capaz de } \\
\text { fazer/realizar } \\
\text { coisas assim como } \\
\text { as demais pessoas }\end{array}$ & $\begin{array}{l}\text { 4. I am able to } \\
\text { do/perform } \\
\text { things as well as } \\
\text { other people }\end{array}$ & $100 \%$ & PA \\
\hline $\begin{array}{l}\text { 5. I feel I do } \\
\text { not have much } \\
\text { to be proud of. }\end{array}$ & $\begin{array}{l}\text { 5. Eu acho que } \\
\text { eu não tenho } \\
\text { muito do que me } \\
\text { orgulhar }\end{array}$ & $\begin{array}{l}\text { 5. I think I do not } \\
\text { have much to be } \\
\text { proud of. }\end{array}$ & $100 \%$ & $\mathrm{PA}$ & $\begin{array}{l}\text { 5. Eu sinto que não } \\
\text { tenho muito do que } \\
\text { me orgulhar }\end{array}$ & $\begin{array}{l}\text { 5. I feel I do not } \\
\text { have much to be } \\
\text { proud of. }\end{array}$ & $100 \%$ & IN \\
\hline $\begin{array}{l}\text { 6. I certainly } \\
\text { feel useless at } \\
\text { times }\end{array}$ & $\begin{array}{l}\text { 6. Às vezes eu } \\
\text { me sinto inútil }\end{array}$ & $\begin{array}{l}\text { 6. Sometimes } \\
\text { I feel myself } \\
\text { useless (worthless) }\end{array}$ & $100 \%$ & PA & $\begin{array}{l}\text { 6. Eu com certeza } \\
\text { me sinto inútil às } \\
\text { vezes }\end{array}$ & $\begin{array}{l}\text { 6. For sure, } \\
\text { I am sometimes } \\
\text { useless }\end{array}$ & $100 \%$ & IN \\
\hline $\begin{array}{l}\text { 7. I feel that } \\
\text { I'm aperson } \\
\text { of worth, at } \\
\text { east on an } \\
\text { equal plane } \\
\text { with others. }\end{array}$ & $\begin{array}{l}\text { 7. Eu sinto que } \\
\text { sou uma pessoa } \\
\text { de valor, no } \\
\text { mínimo tanto } \\
\text { quanto as outras } \\
\text { pessoas }\end{array}$ & $\begin{array}{l}\text { 7. I feel I'm a } \\
\text { valuable person, at } \\
\text { least as valuable as } \\
\text { other people. }\end{array}$ & $100 \%$ & IN & $\begin{array}{l}\text { 7. Eu sinto que sou } \\
\text { uma pessoa de } \\
\text { valor, pelo menos } \\
\text { no mesmo nível do } \\
\text { que as outras }\end{array}$ & $\begin{array}{l}\text { 7. I feel I'm a } \\
\text { valuable person, } \\
\text { at least I am level } \\
\text { with other people. }\end{array}$ & $100 \%$ & IN \\
\hline $\begin{array}{l}\text { 8. I wish I } \\
\text { could have } \\
\text { more respect } \\
\text { for myself }\end{array}$ & $\begin{array}{l}\text { 8. Eu gostaria de } \\
\text { poder ter mais } \\
\text { respeito por mim } \\
\text { mesmo }\end{array}$ & $\begin{array}{l}\text { 8. I would like to } \\
\text { have more respect } \\
\text { for myself }\end{array}$ & $100 \%$ & $\mathrm{PA}$ & $\begin{array}{l}\text { 8. Eu gostaria de } \\
\text { ter mais respeito } \\
\text { por mim mesmo } \\
\text { (a) }\end{array}$ & $\begin{array}{l}\text { 8. I would like to } \\
\text { have more respect } \\
\text { for myself }\end{array}$ & $80 \%$ & PA \\
\hline $\begin{array}{l}\text { 9. All in all, } \\
\text { I am inclined } \\
\text { to feel that } \\
\text { I am a failure }\end{array}$ & $\begin{array}{l}\text { 9. Levando tudo } \\
\text { em conta, eu } \\
\text { penso que eu sou } \\
\text { um fracasso }\end{array}$ & $\begin{array}{l}\text { 9. Taking } \\
\text { everything into } \\
\text { consideration, } \\
\text { I think } \\
\text { I am a failure }\end{array}$ & $50 \%$ & MA & $\begin{array}{l}\text { 9. Resumindo/ No } \\
\text { todo, eu me sinto } \\
\text { inclinada a sentir } \\
\text { que eu sou um } \\
\text { fracasso }\end{array}$ & $\begin{array}{l}\text { 9. To sum up/ As } \\
\text { a whole, I tend to } \\
\text { feel I am a } \\
\text { failure }\end{array}$ & $100 \%$ & IN \\
\hline $\begin{array}{l}\text { 10. I take a } \\
\text { positive } \\
\text { attitude } \\
\text { toward myself }\end{array}$ & $\begin{array}{l}\text { 10. Eu tenho } \\
\text { uma atitude } \\
\text { positiva com } \\
\text { relação a mim } \\
\text { mesmo }\end{array}$ & $\begin{array}{l}\text { 10. I have a } \\
\text { positive attitude } \\
\text { in relation toward } \\
\text { myself }\end{array}$ & $100 \%$ & IN & $\begin{array}{l}\text { 10. Eu tenho/tomo } \\
\text { uma atitude } \\
\text { positiva para } \\
\text { comigo mesmo }\end{array}$ & $\begin{array}{l}\text { 10. I have/take a } \\
\text { positive attitude } \\
\text { toward myself }\end{array}$ & $100 \%$ & IN \\
\hline
\end{tabular}


Quanto a confiabilidade, o alfa de Cronbach obtido foi de 0,68 e o Índice de Correlação Intraclasse (ICC) de 0.7. O kappa variou do regular ao moderado, conforme pode ser observado na Figura 1.

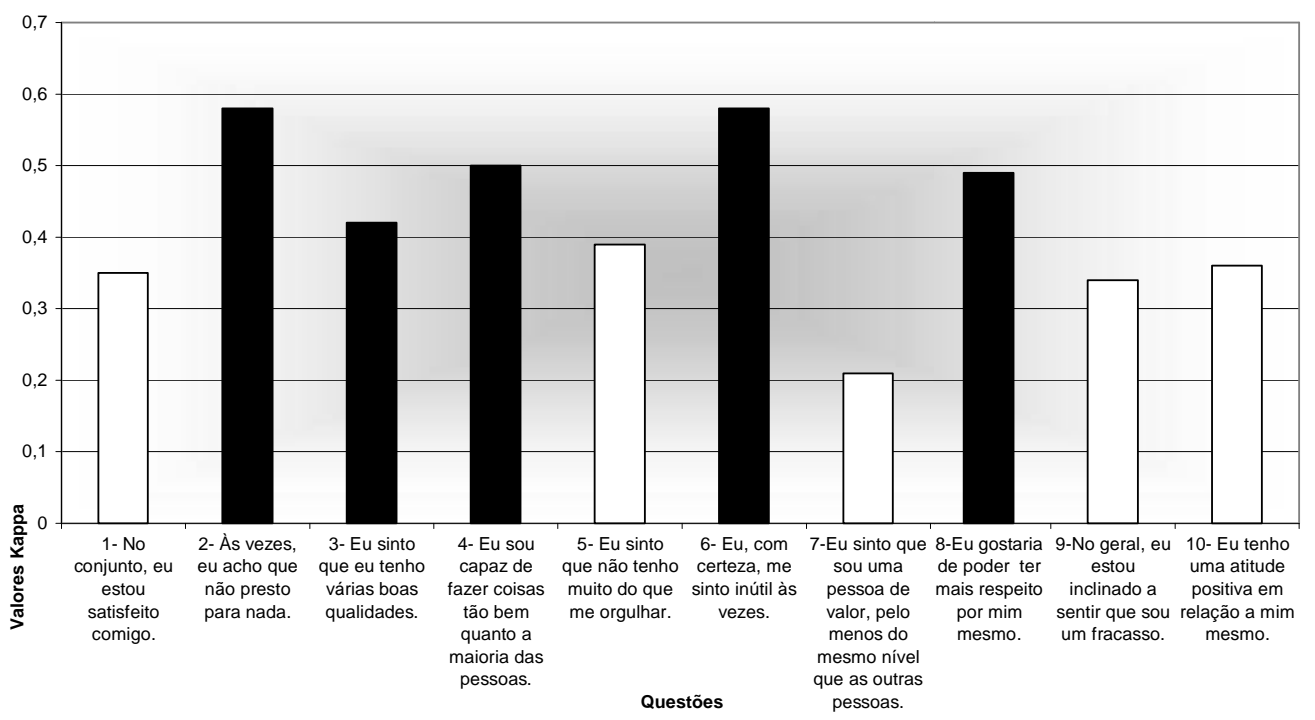

Figura 1. Grau de Concordância da Escala de Auto-Estima de Rosenberg segundo o Kappa Ponderado.

Notas. $\square$ Concordância regular;

Concordância moderada.

No que se refere a análise fatorial, três estruturas de fatores foram geradas, com grau de explicação de $54 \%$ da variância, resultado similar é encontrado na análise do gráfico scree plot, de acordo com os autovalores menores que um. Para uma maior compreensão dos fatores e con- seqüentemente melhor aprimoramento da análise, procedeu-se a rotação ortogonal de eixos varimax normalizada, onde manteve-se o percentual cumulativo e o número de fatores equivalente ao obtido na rotação anterior.

Scree Plot

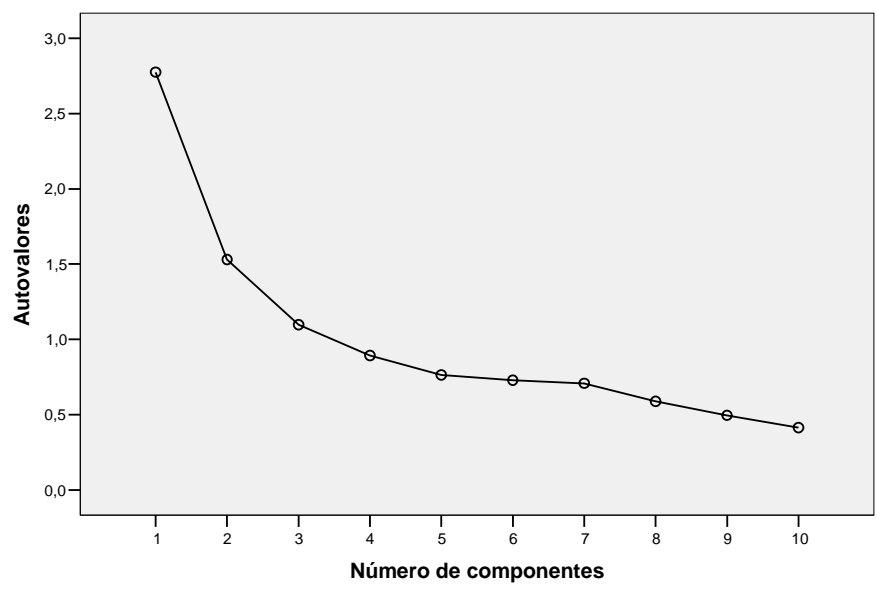

Figura 2. Scree Plot dos Autovalores obtidos na Análise Fatorial da Escala de Auto-Estima de Rosenberg.

Sem detalhar demasiadamente, no primeiro fator concentram-se itens da avaliação negativa de si mesmo, englobando aspectos não valorativos e de sentimento de incompetência pessoal (falta de orgulho por si e/ou por conquistas pessoais, falta de respeito por si e sentimento de fracasso). No fator 2 a avaliação de si é direcionada a um aspecto mais positivo, a saber da percepção de qualidades pessoais e de competência; e no fator 3 mesclam-se itens positivos e negativos de si mesmo, onde os sentimentos pela satisfação ou insatisfação por si mesmo são eviden- 
ciados em termos de valor, competência e sentimento de utilidade na vida. Os itens do terceiro fator também obtiveram cargas fatoriais nos fatores 1 e 2: por exemplo, o item de número 1 (satisfação consigo mesmo) carregou com 0,576 no terceiro fator e 0,406 no segundo fator; os de número 2 e 6 carregaram 0,353 e 0,434 na primeira estrutura fatorial e 0,684 e 0,688 no terceiro fator, respectivamente, condizente a avaliação negativa da estrutura fatorial. Nesses casos, é importante destacar que as cargas fatoriais não divergem consideravelmente das obtidas no terceiro fator.

Sob essa perspectiva, a escala sugere duas estruturas de fatores: (a) fator 1 caracterizado pela baixa auto-estima, que contempla os itens de número 2,5,6,8,9 e (b) fator 2 de al ta auto-estima, que engloba os itens restantes $(1,3,4,7,10)$ - as definições de ambos construtos já foram apresentadas.

Tabela 2

Análise Fatorial da Escala de Auto-Estima de Rosenberg

\begin{tabular}{|c|c|c|c|}
\hline \multirow{2}{*}{$\begin{array}{l}\text { Nível de } \\
\text { Auto-Estima }\end{array}$} & \multirow[t]{2}{*}{ Questões } & \multicolumn{2}{|c|}{ Carga Fatorial } \\
\hline & & Fator 1 & Fator 2 \\
\hline \multirow{5}{*}{ 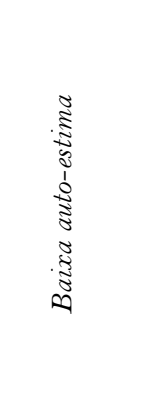 } & $\begin{array}{l}\text { 2- Às vezes, eu acho que } \\
\text { não presto para nada. }\end{array}$ & 0,353 & \\
\hline & $\begin{array}{l}\text { 5- Eu sinto que não tenho } \\
\text { muito do que me orgulhar. }\end{array}$ & 0,678 & \\
\hline & $\begin{array}{l}\text { 6- Eu, com certeza, } \\
\text { me sinto inútil às vezes. }\end{array}$ & 0,434 & \\
\hline & $\begin{array}{l}8 \text {-Eu gostaria de poder ter } \\
\text { mais respeito por mim mesmo. }\end{array}$ & 0,688 & \\
\hline & $\begin{array}{l}\text { 9-No geral, eu estou inclinado } \\
\text { a sentir que sou um fracasso. }\end{array}$ & 0,621 & \\
\hline \multirow{5}{*}{ 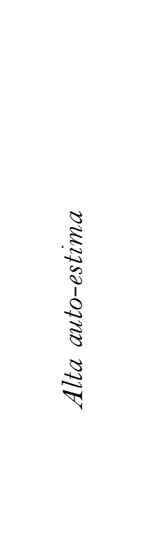 } & $\begin{array}{l}\text { 1- No conjunto, eu estou } \\
\text { satisfeito comigo. }\end{array}$ & & 0,406 \\
\hline & $\begin{array}{l}3-\text { Eu sinto que eu tenho } \\
\text { várias boas qualidades. }\end{array}$ & & 0,529 \\
\hline & $\begin{array}{l}\text { 4- Eu sou capaz de fazer } \\
\text { coisas tão bem quanto } \\
\text { a maioria das pessoas. }\end{array}$ & & 0,697 \\
\hline & $\begin{array}{l}\text { 7-Eu sinto que sou uma } \\
\text { pessoa de valor, pelo menos } \\
\text { do mesmo nível que } \\
\text { as outras pessoas. }\end{array}$ & & 0,666 \\
\hline & $\begin{array}{l}\text { 10-Eu tenho uma atitude } \\
\text { positiva em relação } \\
\text { a mim mesmo. }\end{array}$ & & 0,622 \\
\hline
\end{tabular}

Estudando a validade de construto, constatam-se correlações significativas entre a auto-estima e as escalas estudadas. Na Tabela 3, os coeficientes de correlação de Pearson são apresentados e os respectivos níveis de significância estatística. Constata-se correlação positiva entre os tipos de apoio e auto-estima, ou seja, adolescentes que denotam um sentimento de si mais positivo afirmam ter mais apoio afetivo, emocional, de informação e interação positiva. Com correlação inversa, esses jovens sofrem menos violência psicológica e menos violência entre pais e entre irmãos.
Tabela 3

Coeficientes de Correlação de Pearson da Escala de Auto-Estima de Rosenberg e algumas Variáveis Importantes

\begin{tabular}{|l|c|c|}
\cline { 2 - 3 } \multicolumn{1}{c|}{} & \multicolumn{2}{c|}{ AUTO-ESTIMA } \\
\hline Variáveis & $\begin{array}{l}\text { Coeficiente } \\
\text { de Pearson }\end{array}$ & $\begin{array}{l}\text { Nível de } \\
\text { Significância }\end{array}$ \\
\hline Apoio Afetivo $(N=196)$ & 0,205 & $0,004^{*}$ \\
\hline Apoio Emocional $(N=193)$ & 0,313 & $0,00 *^{*}$ \\
\hline Apoio de Informação $(N=191)$ & 0,239 & $0,001^{*}$ \\
\hline Interação Positiva $(N=179)$ & 0,280 & $0,000^{*}$ \\
\hline Violência Psicológica $(N=174)$ & $-0,292$ & $0,00 *^{*}$ \\
\hline Violência entre Irmãos $(N=168)$ & $-0,160$ & $0,038^{*} *$ \\
\hline Violência Entre Pais $(N=171)$ & $-0,192$ & $0,012^{*} *$ \\
\hline
\end{tabular}

Notas. *Significante a 0,05; **Significante a 0,01.

\section{Discussão}

A importância da auto-estima no estudo de uma série de problemáticas sociais e de saúde exige o enfrentamento de desafios teórico-metodológicos e esse trabalho pode abrir caminhos ou incitar propostas para a discussão da questão no país.

O modelo de adaptação transcultural utilizado foi escolhido por ser abrangente e levar em conta as diferenças do contexto sócio-cultural na adaptação de um instrumento. Embora muitos pesquisadores já estejam preocupados com estratégias de adaptação transcultural de instrumentos criados em uma cultura e transpostos para outra, ainda são escassos estudos que seguem uma metodologia mais refinada. No entanto, esse é um aspecto fundamental que merece destaque na idealização e execução de estudos populacionais, em especial aqueles que envolvem temáticas da área de saúde mental.

A escala em estudo mostra-se eficiente na equivalência funcional, o que indica adequação das equivalências entre a versão original e a proposta, contudo algumas questões devem ser salientadas e ressaltadas.

É clara a apreensão satisfatória da equivalência semântica, tanto no que se refere ao significado geral quanto o referencial. No entanto, as divergências entre as apreciações dos avaliadores, especialmente em relação aos itens 2, 3 e 9 destacam a necessidade da última etapa, onde é feita uma crítica final das etapas anteriores pelos pesquisadores, propondo a versão final.

Como um todo, os resultados dos coeficientes psicométricos encontrados no estudo de confiabilidade e validade do instrumento sugerem a sua aplicação na população estudada. A concordância regular e moderada do Kappa nos itens individuais da escala mostra uma certa fragilidade da escala, o que pode ser relativizado face ao elevado índice de correlação intraclasse (ICC). Conclui-se que a análise mais global do ICC denota a boa confiabilidade da escala como um todo. 
Quanto à consistência interna, o coeficiente alfa diferiu ligeiramente dos obtidos em outros trabalhos, os quais ficaram em torno de 0,8 (Hutz, 2000). Na análise de todos 1686 alunos participantes da pesquisa como um todo, a consistência interna obteve uma ligeira elevação (0.71). Giacomoni (2002) estudando o bem-estar subjetivo de crianças encontrou índice similar (0.75 de Alpha de Cronbach). É também preciso considerar a natureza da amostra desse trabalho, já que é na adolescência que a visão e avaliação de si pode oscilar consideravelmente. Somado a isso, a consistência interna também é afetada pelo reduzido número de itens da escala.

Gonzalez-Fortaleza e Ruiz (1993) adaptando a escala de Rosenberg para adolescentes mexicanos obtiveram alfa de Cronbach de 0.67 e também encontraram três estruturas de fatores numa rotação varimax com grau de explicação de $66,2 \%$ de variância acumulada, com uma disposição diferenciada da encontrada aqui. No entanto, esses autores excluem três itens da versão final, razão provável pela distinta disposição dos itens nas estruturas de fatores.

Os resultados obtidos na avaliação da validade de construto também mostram evidências da adequação da versão proposta, uma vez que as correlações apreciadas foram consistentes com o quadro teórico subjacente. A título de curiosidade, a validade de conteúdo também foi estudada nas etapas iniciais do processo de adaptação da escala, através de discussão teórica com experts da área de saúde mental.

Coadunando com esses resultados de confiabilidade e validade, outro trabalho realizado no município de São Gonçalo/RJ com 1923 adolescentes escolares, no ano de 2003, obteve-se os seguintes resultados na avaliação da presente versão da escala de auto-estima de Rosenberg: alfa de Cronbach de 0.81, ICC de 0.8 e kappa predominantemente moderado. Quanto à validade de construto, a escala de auto-estima correlacionou-se positivamente com todos os tipos de apoio e satisfação de vida, e inversamente com sofrimento psíquico e violência psicológica (Assis, Pesce \& Avanci, 2006). Esses achados ratificam a consistência dos resultados obtidos e reforçam a utilização da escala, que segue sendo o instrumento mais indicado e utilizado em contexto de investigação para avaliação da auto-estima (Dukes \& Martinez, 1994; Hutz, 2000).

O formato e a forma de aplicação auto-preenchível também foi satisfatória, mantendo-se o mesmo número de questões e as mesmas opções de respostas que o instrumento original. É importante salientar que um profissional treinado deve acompanhar a aplicação da escala. O instrumento obteve plena aceitação e compreensão na população, com baixo percentual de itens deixados em branco e sem recusa. Esse resultado é um aditivo importante para a utilização do instrumento e que responde à avaliação da equivalência operacional.

Embora alguns trabalhos nacionais (Giacomoni, 2002; Gobitta, 2000; Hutz, 2000) possam dialogar com os achados aqui apresentados, a divergência metodológica e a utilização de diferentes instrumentos e versões impossibilitam comparações, o que representa uma limitação ao tra- balho. Por exemplo, Gobitta (2000), com uma proposta diferenciada, realizou a adaptação transcultural da escala de auto-estima de Coopersmith (1967), instrumento com uma abordagem da auto-estima em áreas relacionais específicas da vida, onde é avaliado o sentimento de si, em termos valorativos e de competência, na família e na escola, por exemplo. Essa abordagem também parece adequada em estudos com objetivos mais específicos e direcionados.

No entanto, para o estudo da auto-estima na infância e adolescência, Dukes e Martinez (1994) ressaltam que a percepção de si mais global, o autoconceito, é predominante nessas fases da vida, o que parece enfraquecer na vida adulta, época em que as competências se tornam mais definidas e diferenciadas. O autoconceito está diretamente relacionado à auto-estima, havendo muita dificuldade de delimitação clara entre esses dois construtos (Gobitta, 2000; Mruk, 1995).

Sem dúvida, que o estudo da auto-estima permeia complexidades e contradições, já que questões culturais, psicológicas e individuais perpassam esse sentimento positivo e negativo de si mesmo. Contudo, ainda que os especialistas tenham considerado todos os itens da escala pertinentes ao contexto (em especial à população escolar adolescente), sugere-se a necessidade de aplicação dessa versão em português em amostras diferenciadas, por exemplo, em indivíduos adultos e não escolares, tal como é usado em inúmeros trabalhos internacionais (Lam \& Grossman, 1997; Silverstone \& Salsali, 2003); a fim de verificar a prevalência, a confiabilidade e a validade do instrumento. Ajustes locais e regionais também podem se fazer necessários, em face da diversidade cultural brasileira. Certamente, que essas informações seriam enriquecedoras para o aprimoramento de estudos sobre a auto-estima em âmbito nacional, o que contribuiria em aspectos relevantes para a promoção da saúde mental.

Uma outra consideração importante, é que a escala de auto-estima de Rosenberg foi estudada numa população não clínica e para fins de pesquisa, não sendo possível utilizá-la para diagnóstico, mas sim para rastreamento do sentimento de si (auto-estima) na população geral. Não há conhecimento de sua eficiência na clínica.

O esclarecimento da magnitude da auto-estima em diversos contextos possibilita o conhecimento de um 'atributo' importante na área de saúde mental e coletiva, o que pode ser uma importante estratégia para a prevenção de problemas no crescimento e desenvolvimento de crianças e adolescentes. É um desafio apreender sobre mecanismos que protegem um indivíduo do risco, como é o caso de uma elevada auto-estima, e inseri-los como prioridade na implantação de programas de prevenção primária, promoção de saúde e de bem estar psicológico.

Enfim, esse trabalho é bastante pertinente para o estudo da auto-estima e para a área da avaliação psicológica, uma vez que apresenta e propõe estratégias plausíveis e adequadas ao desenvolvimento e adaptação de instrumentos em psicologia. Mesmo tendo sido encontradas limitações, elas devem servir de estímulo a outros pesquisadores que queiram enfrentar o desafio das técnicas estatísti- 
cas e de alguns temas caros à psicologia e à avaliação psicométrica, entendendo que a apropriação desse instrumental é fundamental para o desenvolvimento do conhecimento de clínicos e pesquisadores.

\section{Referências}

Assis, S. G., \& Avanci, J. Q. (2003). Labirinto de espelhos. A formação da auto-estima na infância e adolescência. Rio de Janeiro, RJ: Editora Fiocruz.

Assis, S. G., Pesce, R. P., \& Avanci, J. Q. (2006). Resiliência. Enfatizando a proteção na adolescência. Porto Alegre, RS: Artmed.

Avanci, J. Q., Assis, S. G., Santos, N. C. dos, \& Oliveira, R. V. C. (2005). Escala de violência psicológica contra adolescentes. Revista de Saúde Pública, 39(5), 702-8.

Branden, N. (2000). Auto-estima: Como aprender a gostar de si mesmo. São Paulo, SP: Saraiva

Baumeister, R. F., Smart, L., \& Boden, J. M. (1996). Relation of threatened egotism to violence and aggression: The dark side of high self-esteem. Psychological Review, 103(1), 5-33.

Chor, D., Griep, R. H., Lopes, C. S., \& Farstein, E. (2001). Medidas de rede e apoio social no estudo pró-saúde: Pré-testes e estudo piloto. Cadernos de Saúde Pública, 17(4), 887-896.

Coopersmith, S. (1967). The antecedents of self-esteem. San Francisco, CA: Freeman

Dourado, J. M. B. (1984). O rendimento acadêmico e sua relação com o autoconceito do aluno e a retroalimentação do professor. Disser tação de Mestrado não-publicada, Universidade de Brasília, DF.

Dukes, R. L., \& Martinez, R. (1994). The impact of ehgender on self-esteem among adolescents. Adolescence, 29(113), 105115 .

Giacomoni, C. H. (2002). Bem-estar subjetivo infantil: Conceito de felicidade e construção de instrumentos para avaliação. Tese de doutorado não-publicada, Curso de Pós-graduação em Psicologia do Desenvolvimento, Universidade Federal do Rio Grande do Sul, Porto Alegre, RS.

Gobitta, M. (2000). Estudo inicial do inventário de auto-estimaForma A. Dissertação de Mestrado não-publicada, Instituto de Psicologia e Fonoaudiologia, Pontifícia Universidade Católica de Campinas, SP.

Gomes, R. A. (1994). Violência enquanto agravo à saúde de meninas que vivem nas ruas. Cadernos de Saúde Pública, 1O(1), 156-167.

Gonzalez-Fortaleza, C. F., \& Ruiz, E. R. (1993). Autoestima y sintomatología depresiva en adolescentes mexicanos: un estudio correlacional por género. Revista ABP-APAL, 15(2), 59-64.

Hasselmann, M. H., \& Reichenheim, M. E. (2003). Adaptação transcultural da versão em por tuguês da conflict tactics scales R (CTS-1), usada para aferir violência no casal: Equivalências semântica e de mensuração. Cadernos de Saúde Pública, 19(4), 1083-1093.

Herdman, M., Fox- Rushby, J., \& Badia, X. (1998). A model of equivalence in the cultural adaptation of HRQol Instruments: The universalist approach. Quality of Life Research, 4, 323335 .

Hutz, C. S. (2000). Adaptação brasileira da escala de auto-estima de Rosenberg: Curso de Pós-Graduação em Psicologia do Desenvolvimento, Universidade Federal do Rio Grande do Sul, Porto Alegre, RS. Mimeo.
Kaplan, S. J., Pelcovitz, D., \& Labruna, V. (1999). Child and adolescent abuse and neglect research: A review of the past 10 years. Journal of the American Academy of Child \& Adolescent Psychiatry, 38(10), 1214-1222.

Lam, J. N., \& Grossman, F. K. (1997). Resiliency and adult adaptation in women with and without self-reported histories of childhood sexual abuse. Journal of Traumatic Stress, 10(2), 175-196.

Mecca, A., Smelser, N. J., \& Vasconcellos, J. (1989). The social importance of self-esteem. Berkeley, CA: University of California Press.

Moraes, C. L. (2001). Aspectos metodológicos relacionados a um estudo sobre a violência familiar durante a gestação como fator de propensão da prematuridade do recém-nascido. Tese de Doutorado não-publicada, Curso de Pós-Graduação em Ciências, Escola Nacional de Saúde Pública, Fundação Oswaldo Cruz, Rio de Janeiro, RJ.

Moraes, C. L, \& Reichenheim, M. E. (2002). Cross-cultural measurement equivalence of the revised conflict tactics scales (CTS2). Portuguese version used to identify violence within couples. Cadernos de Saúde Pública, 18, 783-796.

Mruk, C. (1995). Self-esteem: Research, theory, and practice. New York: Springer.

Roberts, S. B., \& Kendler, K. (1999). Neuroticism and self-esteem as indices of the vulnerability to major depression in women. Psychological Medicine, 29, 1101-1109

Rosenberg, M. (1989). Society and the adolescent self-image. Princeton, NJ: Princeton University Press. (Original publicado em 1956)

Silverstone, P. H., \& Salsali, M. (2003). Low self-esteem and psychiatric patients: Part II - The relationship between selfesteem and demographic factors and psychosocial stressors in psychiatric patients. Annals of General Hospital Psychiatry, 2,3 .

Shrout, P. E. (1998). Measurement reliability and agreement in psychiatriy. Statistical methods in medical research, 7, 301-317.

Streiner, D. L., \& Norman, G. R. (1995). Health measurement scales. A practical guide to their development and use. Oxford, UK: Oxford University Press

Souza, D. B. L. de, \& Ferreira, M. C. (2005). Auto-estima pessoal e coletiva em mães e não-mães. Psicologia em Estudo, 1O(1), 19-25.

Tamayo, A., \& Cunha, P. (1983). Autoconceito, sexo e frequiência de atividade sexual pré-marital. Ciência e Cultura, 35(7).
Recebido: 09/09/2005 $1^{a}$ revisão: 20/10/2006 $2^{a}$ revisão: $11 / 12 / 2006$ Aceite final: 19/12/2006 